Letras, Lima 48 (84-85): 84-110, 1976.

\title{
El conocimiento del espacio geométrico euclidiano
}

GUSTAVO SACO

\begin{abstract}
El presente trabajo indaga los diversos modos cómo el espacio físico determina las propiedades geométricas y cómo este mismo espacio es el que nos proporciona las bases para llegar a su conocimiento. El conocimiento adquirido a través del auscultamiento de ese espacio físico constituye la piedra de toque decisiva sobre la cual se desarrolla la geometría. La tridimensionalidad del espacio que siglos de cultura y de experiencias con la naturaleza no han logrado rebasar este marco, explican la consistencia de los conocimientos Jgeométricós.el ELcontinuum cuatridimensional de la teoría de la relatividad no anula la tridimensionalidad del espacio sino que la armoniza con la unidimensionalidad del tiempo. Por otro lado, el espacio de ni-dimensiones de la ciencia contemporánea no son sino abstracciones matemáticas, y no hay alguna prueba de que el espacio físico se comporte de esa manera. Tampoco es reducible la geometría a las relaciones lógicas, aun cuando la lógica es un poderoso procedimiento en el desarrollo de la geometría. Si se intenta reducirla, como lo pretenden algunos matemáticos, es porque se está utilizando un mismo término, geometría, para referirse a dos disciplinas, que se complementan pero que no son identificables.
\end{abstract}

Empirismo geométrico y observación numérica concreta

El conocimiento espontáneo e inmediato de las propiedades tridimensionales del espacio, se amplía como lo hicieron los antiguos egipcios y babilonios al hallar diversas relaciones en el es- 
pacio por medio de figuras geométricas, empíricamente diseñadas. No obstante que estudiaron sólo de manera empírica algunas relaciones de magnitud de muy pocas figuras geométricas, como el triángulo, el círculo, la esfera, la pirámide, el cuadrilátero y los ángulos, llevados por sus necesidades fundamentales de medir el suelo, y por sus construcciones, dieron un paso decisivo para trascender los límites del conocimiento inmediato y espontáneo del espacio. Fue evidente, a partir de aquella época, que dentro de la tridimensionalidad del espacio, era posible analizar otras propiedades que hubieran permanecido desconocidas, a no ser por las necesidades de medición. Es cierto también que al conocimiento de las áreas y volúmenes solamente llegaron aproximadamente, aún cuando fueron más exactos al establecer la relación entre la circunferencia y el diámetro, y las relaciones entre los lados de un triángulo. En ningún caso lograron demostrar de un modo deductivo la validez de las propiedades de las figuras geométricas. Sin embargo, aún cuando no demostraron, por lo menos mostraron esas propiedades, sin alcanzar, por supuesto, la abstracción y generalización que iba a alcanzar posteriormente la geometría de los griegos. No obstante, el procedimiento empírico de los egipcios y babilonios revela que no es indispensable que, para conocer las propiedades geométricas, sea necesaria la demostración a través de la deducción, partiendo de ciertos principios. El procedimiento empírico en el tratamiento de las figuras geométricas nos da a conocer ciertas propiedades de las figuras, así como el procedimiento experimental en la física, aún cuando no sean tan decisivas ni concluyentes, por no partir de principios y deducciones a base de esos principios. De igual modo, la geometría empírica, si no hubiese sido utilizada la demostración deductiva, hubiese proporcionado conocimientos semejantes a los que ha revelado la física, cuando innumerables investigadores se hubiesen dedicado a estudiar, sobre la base exclusiva de la experiencia, las propiedades de las figuras geométricas, pero no poseería la evidencia generalizadora, como tampoco la posee la física del presente. Por lo demás la topología o analysis situs en algún momento procede basándose en la experiencia, pues se descubren ciertas relaciones espaciales sin partir de principios, la cual nos indica el camino que hubiese seguido la geometría de no mediar la demostración deductiva, en tanto que indica también que no es forzoso el dejarse llevar por la demostración deductiva como el único procedimiento para conocer las relaciones espaciales de las figuras geométricas. Es un hecho histórico que el conocimiento de las relaciones espaciales, a través de la geometría empírica, de los egipcios y babilonios, durante siglos no sobrepasó el estadio rudimentario; pero igual consideración se puede hacer en otras áreas del saber como la astronomía y el conocimiento de la naturaleza, a 
pesar que ya tuvieron conocimiento rudimentarios también en estas áreas. Son los factores históricos y sociales los que permitieron que solamente a un ritmo muy lento se desarrollara el conocimiento en cualquier área. De ningún modo es justificado responsabilizar a la ausencia del método deductivo, el estado rudimentario de la geometría entre los egipcios y babilonios, sino exclusivamente a su insuficiente desarrollo social, lo que repercutió no solamente en lo rudimentario de la geometría sino también en otras disciplinas.

La geometría bien pudo haberse desenvuelto a través de los siglos como una disciplina basada en la experiencia, como se desenvolvieron otras disciplinas, y no habría que distinguir entre geometría física o aplicada, por un lado, y geometría matemática o pura, por otro. Su suerte hubiese sido la misma que la que siguieron las ciencias de la naturaleza.

Como las necesidades de medición del suelo y de la construcción llevó a los egipcios a un primer conocimiento empírico de la geometría, sin embargo, estos conocimientos tan elementales posibilitaron el advenimiento de la geometría de Tales de Mileto. En efecto, por los relatos históricos se sabe que Tales viajó a Egipto y tuvo conocimiento de la geometría elemental egipcia.

\section{Observación y deducción geométricas}

A los griegos se les atribuye el descubrimiento del procedimiento lógico y deductivo ên la geometría y on Tales de Mileto el haber iniciado la nueva era de.la geometría. Aún cuando ha quedado únicamente para la posteridad el enunciado de pocos teoremas sobre ángulos, triángulos y círculos, no ha quedado consignada la demostración que pudo haber tenido para esos teoremas. Sin embargo, el mismo enunciado de los teoremas revela un poder de generalización que difiere fundamentalmente de los datos numéricos y cuantitativos de las relaciones espaciales de las figuras geométricas de los egipcios y babilonios. No porque a estos últimos les haya faltado la generalización, como se constata en sus enunciados sobre el volumen y las áreas de las figuras geométricas que estudiaron, sino que su generalización era equivocada por cuanto no expresaba la verdadera relación espacial, o porque tan sólo expresaba ciertas relaciones numéricas de las relaciones que habían descubierto. En cambio, en Tales de Mileto se constata un enunciado de los teoremas hecho de un modo tan general, cuyo nivel de abstracción deja de lado toda referencia numérica o cuantitativa, así como el enunciado de sus teoremas expresa tan fielmente la relación geométrica, que hoy mismo podemos comprobar su verdad. Es de suponerse que Tales para llegar a enunciados 
tan generales de los teoremas geométricos, haya tenido que demostrar su validez, aun cuando no conozcamos qué demostración utilizó. Si esta es la situación preguntémonos qué tipo de demostración pudo haber utilizado, para lo cual tomemos en cuenta los diversos teoremas que se le atribuyen.

El estudio de las proposiciones geométricas de Tales revela que es prácticamente suficiente observar las figuras geométricas a las que se refieren sus proposiciones para tener la evidencia de la verdad del enunciado acerca de las figuras. Así en las proposiciones: Un círculo se corta en dos por cualquier diámetro; los óngulos de la base de un trióngulo isósceles son iguales; si dos líneas rectas se cortan los óngulos opuestos son iguales (o los ángulos verticales formados por dos líneas rectas que se cortan son iguales). Estas tres proposiciones geométricas permiten constatar que su conocimiento requiere únicamente capacidad intelectual de observar las características de una figura geométrica para tener el conocimiento de esas características, sin haber mediado ninguna demostración o deducción lógica propiamente.

La proposición geométrica de Tales: El ángulo inscrito en un semicírculo es un ángulo recto, proposición que fue conocida por los babilonios como un hecho geométrico, hace ver, como seguramente lo hicieron los babilonios, que el cuscultamiento de la figura correspondiente, permite por la simple observación, tener evidencia de la verdad de esta proposición, por lo cual, tampoco haría falta una demostración lógica propiamente, o por lo menos sitúa a esta proposición entre un camino intermedio entre la simple observación, como las otras trescproposicionesemencionadas anteriormente. En todo casor no es indispensable una demostración lógica, para tener la convicción de su verdad. Otras proposiciones geométricas atribuidas a Tales como la que enuncia que dos triángulos son congruentes si tienen dos ángulos y un lado respectivamente iguales, pueden también comprobarse con una relativamente sencilla construcción de dos trióngulos, y constatar que se superponen adecuadamente. En cambio, el teorema llamado propiamente de Tales, y que se anuncia de la siguiente manera: los lados de ángulos iguales en los triángulos semejantes son proporcionales, requiere, efectivamente, en este caso, de una verdadera comparación de dos triángulos y establecer mediante la utilización de razones geométricas, la proporcionalidad de los lados. Es tam. bién cierto que con esta comparación se tiene un conocimiento adecuado de la verdad de una proposición geométrica, sin necesidad cuún de llegar a la demostración. La historia nos dice que este teorema fue utilizado por Tales para medir la altura de la gran pirámide de Cheops. Digamos que en este último teorema no es suficiente una simple cuscultación u observación de la figura geométrica y requiere, por lo menos, comprobar por medio de razones 
elementales geométricas, la verdad de la proposición, comprobación es que ya una demostración, pero todavía no es una demostración lógica deductiva, a partir de axiomas geométricos fundamentales. ¿Llegó Tales a demostrar de manera lógico-deductiva la verdad de las seis proposiciones geométricas que se le atribuyen? No ha quedado en la historia nada que confirme que utilizó este tipo de demostración. En cambio, nos queda el firme convencimiento, basándonos en las proposiciones geométricas que ha dejado, que utilizó la generalización en las verdades geométricas, lo cual implicaba un vuelco completo en el espíritu de la ciencia.

Es verdad que todas las proposiciones geométricas de Tales de Mileto pueden ser demostradas de un modo lógico-deductivo, pero el análisis de esas proposiciones nos hace ver que es suficiente una abstracción generalizadora, una decisión del intelecto, que va a ser verificada en todos los casos en que la mente tenga que enfrentarse a las figuras geométricas. Dos pasos ha dado ya el conocimiento de las relaciones espaciales, como se reflejan en las figuras geométricas: 19 La geometría empírica de los egipcios y babilonios, sobre la base de la observación de las relaciones numéricas, cuantitativas; $2^{\circ}$ La geometría generalizadora de Tales de Mileto sobre la base de la observación de las relaciones espaciales de las figuras geométricas. El tercer paso, el lógico-deductivo, lo hallaremos al llegar a la escuela pitagórica. De todos modos, la geometría de los egipcios y babilonios y la de Tales de Mileto, nos ha enscñado la existencia de dos momentos en el conocimiento geométrico: La observación numérica concreta; y observación abstracta y generalizadora; igualmente nos ha enseñado que para el conocimiento de algunas propiedades geométricas elementales es únicamente suficiente la observación de las relaciones numéricas que se descubren en las partes de una figura, o la observación de una figura misma, auscultando la relación que haya entre sus partes, y generalizando posteriormente estas relaciones. La generalización geométrica no tiene por qué seguir otro camino fundamentalmente diferente al que sigue la generalización en el conocimiento de la naturaleza física 0 social. De aquí se desprende que la geometría no ha de partir necesariamente de axiomas, y deducir, partiendo de los axiomas, las propiedades de las figuras geométricas. Solamente podrá establecerse que el conocimiento partiendo de los axiomas lleva a una mayor profundidad, a una mayor evidencia y ordenamiento de las verdades geométricas, pero que no es forzosamente el único, pues para un grado de avanzado del conocimiento, es suficiente la abstracción generalizadora, erigida sobre la base de la observación. Queda sin embargo pendiente la cuestión de que da la abstracción generalizadora a la geometría tal carácter de evidencia que no parecen 
poseer las generalizaciones en otras áreas que no sean matemáticoss.

\section{Geometría demostrativa}

Con la escuela pitagórica la geometría alcanza definitivamente el nivel de demostración de los teoremas. Aun cuando son muy contados los teoremas geométricos que se atribuye a los pitagóricos, la demostración que elaboraron para esos teoremas consagra de manera decisiva una nueva orientación en el estudio de la geometría. Así, el teorema fundamental de la geometría, conocido como el teorema de Pitágoras, fue demostrado por los pitagóricos, de modo convincente. No obstante, el modo de demostración que se supone que fue utilizado en este teorema y que se consigna todavía en Euclides, revela trazas de un modo empírico de demostración. Primeramente, en cuanto a su origen, las antiguas culturas orientales conocieron empiricamente la existencia de un triángulo en el cual uno de sus lados debería de mantener cierta definida proporción como de 5,4 y 3 , como ya lo conocieron los egipcios y aun también los babilonios. En segundo lugar, un tipo de demostración consiste en trazar un cuadrado a partir de la hipotenusa, y a cada uno de los catetos, y argumentar que la figura cuadrada tendida sobre la hipotenusa es igual a las figuras cuadradas tendidas sobre cada uno de los catetos. Es un tipo de demostración que utiliza la comparación de figuras y que de un modo visualiza esta relación entre elcuadrado de la hipotenusa y el de los catetos. Subdividiendo a cada una de las figuras cuadradas en pequeños cuádrados iguales todos ellos entre sí, con sólo contar estos pequeños cuadrados podemos constatar que la figura cuadrada tendida sobre la hipotenusa es igual a la suma de las figuras cuadradas tendidas sobre cada una de los catetos. En una primera instancia es entonces posible llegar a un tipo de demostración que prácticamente, de un modo empírico, permita constatar la verdad de la proposición geométrica, y que posiblemente ha sido así conocido por los antiguos egipcios y babilonios. Esta verdad geométrica que vendría a ser posteriormente el teorema de Pitágoras, y que es uno de los teoremas fundamentales de la geometría, pudo haber sido conocido sobre la base empírica, sin poseer, por supuesto, el grado de generalización que va a adquirir con los mismos pitagóricos. Los orígenes del teorema de Pitágoras constituyen una comprobación definitiva que la verdad de las proposiciones geométricas no requiere necesariamente de una demostración lógico-deductivo, pues es suficiente una demostración empírica y a partir de la cual, se puede hacer una abstracción generalizadora. Es muy probable que el destino de muchas otras 
proposiciones hubiese sido el mismo, a no ser por la elaboración sistemática del procedimiento lógico-deductivo con Euclides especialmente. La demostración atribuida propiamente a los pitagóricos, inscribiendo un cuadrado dentro de otro mayor, y deducir la verdad del teorema a base de los triángulos rectángulos que se formon en el interior, sí responde a una demostración lógico-deductiva, con lo cual se inicia un procedimiento esencialmente diferente que probablemente tuvo sus comienzos en Tales de Mileto.

Otro teorema fundamental atribuido a los pitagóricos por Eudemo es el concerniente a la suma de los ángulos de un triángulo. Los pitagóricos lo demostraron trazando a partir del vértice de un ángulo, una paralela al lado considerado como la base del triángulo. La demostración precisa y clara de este teorema revela también que los pitagóricos se hallaban ya alejados de las comprobaciones empíricas, o de una simple observación de la figura geométrica misma, para inducir sus conclusiones; y aún más, esta demostración incluye el concepto de paralelas. Pero este concepto de las paralelas, a atenernos específicamente al teorema, no conlleva todavía la evidencia que va a poseer posteriormente el postulado de las paralelas que como quinto postulado se enuncia por Euclides. Esto significa que la suma de los ángulos de un triángulo puede demostrarse partiendo únicamente de un concepto de paralelas, pero sin que se requiera forzosa o necesariamente, deducir la verdad del teorema, de modo lógico, partiendo de un postulado. La demostración pitagórica no alcanza el grado de generalización lógica que va a tener posteriormente por su relación con el quinto postulado. I La misma demostración pitagórica permite constatar que no es esencial para un primer conocimiento de la verdad de un teorema de la geometría, el que tenga que derivarse de un postulado.

\section{Geometría y coincidencia}

Al iniciar el estudio de la geometría de Euclides nos encontramos con sus definiciones, postulados y nociones comunes (llamados los dos últimos posteriormente axiomas) de los cuales ha de deducirse, como última instancia, las propiedades enunciadas por los teoremas. ¿En qué se justifica Euclides para establecer sus postulados y nociones comunes y dar a las definiciones el significado que les fue dado? La respuesta clásica es que los postulados y las nociones comunes (axiomas) son evidentes por sí mismas y por lo tanto no tienen demostración, y que las definiciones tienen o expresan la cualidad esencial de las propiedades geométricas. Sin embargo, encontramos que las definiciones no van acompañadas de ninguna demostración, por lo cual creemos que 
es justificado situarlos gnoseológicamente al lado de los postulados y axiomas de la geometría euclidiana, puesto que no hay ninguna argumentación que nos lleve a aceptar las definiciones y por lo que aparecen también como evidentes por sí mismas, no requiriendo demostración alguna. Esto último da una característica semejante a los postulados, nociones comunes y definiciones de la geometría euclidiana. Averigüemos si esta aparente similitud es en el fondo esencialmente justificada. Pero, previamente tenemos que preguntarnos por qué los postulados y las nociones comunes son verdades evidentes por sí mismas que no requieren demostración. Comencemos por las nociones comunes.

El término nociones comunes utilizado por Euclides nos indica que no tienen validez únicamente referidas a las propiedades geométricas, sino que también valen para otra propiedad que encierra alguna magnitud, como se desprende del enunciado de las mismas nociones comunes y entre las cuales consideremos las siguientes: Las cosas que son iguales a la misma cosa son también iguales entre sí; Si iguales son añadidos a iguales todos son iguales; $\mathrm{Si}$ iguales son sustraídas de iguales, la resta es iguali Las cosas que coinciden una con la otra son iguales la una con la otra; El todo es mayor que la parte. ¿Por qué estas nociones comunes son evidentes por sí mismas y no requieren demostración? La geometría y la filosofía clásica se han conformado con esta constatación, pero es nuestro intento encontrar una explicación a estas verdades evidentes $\mathrm{y}$ a su indemostrabilidad.

Observemos que las nociones comunes: Las cosas que son iguales a la misma cosa son también iguales entre si; y la que enuncia que las cosas que coinciden la una con la otra son iguales entre sí, son axiomas de un mismo tipo y con rigor lógico deberíamos poner más bien como primer axioma aquél que nos indica el criterio de igualdad, que es el de la colncidencia, antes de proceder a referir a las cosas que son iguales a una misma cosa. Por este motivo, invirtiendo el orden de los axiomas, debe ir en primer lugar el que se refiere a que las cosas que coinciden son iguales la una con la otra. Por lo mismo, este último axioma debe anteceder a aquellos dos que se refieren $\alpha$ iguales que son añadidos o restados que son iguales. Procedamos entonces a considerarlos en un orden diferente a como los expone Euclides en sus Elementos. La noción común que enuncia que las cosas que coinciden la una con la otra son iguales la una a la otra, al poner el acento en la coincidencia, sin especificar en qué consiste, pues que se refiere a un concepto muy general, hace necesario la especificación para indagar cuál es la fuente del conocimiento que posibilita el concepto de la coincidencia. La coincidencia es un concepto recogido en Ia experiencia del mundo físico, cuando al constatar que dos de sus objetos presentes ante la observación son po- 
seedores de rasgos o características que se superponen. Con la aparición del concepto de coincidencia se halla la percepción de semejanza de los objetos físicos, lo cual es un conocimiento que se deriva de la experiencia. En el proceso del conocimiento se da primeramente la percepción de coincidencia de semejanza de los objetos físicos a lo cual sigue posteriormente el concepto de igualdad de esos mismos objetos físicos, concepto de igualdad al cuai se llega por una reflexión sobre la misma percepción de coincidencia y de semejanza. Esta coincidencia a su vez, se debe a que los objetos físicos mismos son poseedores de cualidades que son las mismas, por haber una causa física que así los ha producido. Los niños también tienen la noción de parecido, que es su forma de expresarse sobre las cosas que los adultos llaman semejantes, y que se les manifiesta en su auscultación del mundo físico, en el asunto que ahora estamos tratando. Posteriormente surge en ellos la noción de cosas iguales que se apoya en estas primeras experiencias de cosas coincidente, parecidas o semejantes. En un nivel más alto la noción de coincidencia, se debe a un afinamiento de la igualdad y exige una mayor elaboración mental, puesto que destaca cosas que son iguales en todas sus características, a lo cual se llega por un complejo proceso de análisis y de síntesis de los objetos físicos considerados. Digamos que en este proceso de abstracción se ve la posibilidad que tiene el pensamiento de pasar de la coincidencia y de la semejanza percibida de los objetos físicos a la constatación de su igualdad. Independientemente de los términos que se utilice es conveniente, sin embargo, precisar que en este aspecto del lconocimiento hay tres niveles que para clari. dad conviene denominar, percepción de coincidencia, de semejanzas y constatación de la igualdad.

\section{Coincidencia geométrica y física}

No obstante, expresados de un modo general los conceptos de semejanza, igualdad y coincidencia no explican las diferencias que puede haber entre estos tres, pues en cierto modo los tres obedecen a un mismo proceso conceptual pues lo igual coincide, o lo semejante es igual, o lo que coincide es semejante. Lo importante es entonces destacar el origen de estos conceptos. Así, el concepto de coincidencia es el que mejor se presta para reflejar los orígenes de la relación física entre diversos objetos; el concepto de semejanza, las características comunes entre los mismos objetos; y el concepto de igualdad, para expresar hasta sus niveles más abstractos cualquier concordancia entre entes exclusivamente físicos, o entre entes puramente conceptuales o ideales. Por otro lado, la dificultad para establecer una diferencia radical entre los 
tres conceptos, revela su comunidad de origen. Efectivamente, si bien los estímulos físicos perceptivos tienen necesariamente su origen en el mundo físico, al estimular lo físico los sentidos, pone en juego $y$ en actividad las posibilidades psicológicas conceptuales. Esto es así definitivamente en el adulto, pues si este último superpone dos objetos físicos en los cuales todas sus partes encajan la una con la otra, el adulto no se limita a constatar la coincidencia física entre los dos, sino que en él se pone en actividad el concepto de semejanza $y$ el de igualdad. En el adulto, la coincidencia física desencadena de modo casi inmediato, el concepto de semejanza y el de igualdad. Pero la coincidencia física tiene dos niveles, el primero de los cuales se manifiesta con los objetos que son percibidos tosca y rudimentariamente, $y$ el segundo de modo muy elaborado en el cual se compara muy minuciosamente las partes de los objetos y en el cual se pone en juego un concepto claro y distinto de la igualdad. El primer nivel es propio de los niños y el segundo propio de los adultos. Es en los adultos en donde se dificulta diferenciar la coincidencia física del concepto elaborado de igualdad y semejanza, en tanto que en el niño, mientras más pequeño es, tanto más se halla la coincidencia física del nivel abstracto conceptual. Por lo anterior, se comprueba también que la coincidencia física y su concepto rudimentorio precede al nivel elaborado de coincidencia $\bar{y}$ de igualdad. Ahora bien, si por un lado, la coincidencia física precede al concepto elaborado de igualdad, por otro lado, el parecido físico de los objetos que de un modo tosco y rudimentario se da en los niños, se halla alejado del concepto de semejanza ide los adultos? Y tanto más cuanto más pequeño es el niño. Pues bien así como la coincidencia física rudimentaria da origen "al concepto de igualdad de los adultos, así también el parecido físico de los objetos es la otra fuente del concepto de igualdad en los adultos. De aquí que la coincidencia física y el parecido físico de los objeto sea anterior al de igualdad.

Por las anteriores consideraciones comprabamos que en las nociones comunes (axiomas) como lo planteó la geometría de Euclides, si bien para el adulto que las enuncia se le presentan como verdades válidas por sí mismas, que no requieren demostración, sin embargo, tienen una profunda base en la experiencia del individuo en su contacto con el mundo físico exterior, lo cual cuando menos permite explicar su origen empírico y de aquí que estos axiomas evidentes, sean explicables. En primer lugar, el axioma que enuncia que las cosas que coinciden la una con la otra son iguales la una a la otra, y por lo cual este axioma que se presenta como evidente por sí mismo, es el resultado de innumerables experiencias. Por de pronto, si bien no puede ser aún demostrado, es explicable en su fuente de la experiencia y en su descrrollo psicológico. Pero de esta misma explicación de la convicción psi- 
cológica de la igualdad, es al mismo tiempo, una demostración si llegamos a la fuente que ha originado el postulado. Porque justamente, yuxtaponiendo un objeto físico sobre otro y constatando que se ajustan en todas sus partes, es que podemos demostrar que son iguales, y si no se ajustan, demostramos que no son iguales. En lo que acontece en el mundo físico, esto es definitivamente una demostración que se basa en la naturaleza y en el comportamiento de los objetos físicos mismos. Por lo cual se puede aseverar que el postulado de la coincidencia se demuestra observando los objetos del mundo físico. El postulado de la coincidencia es fundamentalmente un postulado de la física. Sin embargo, ¿por qué es evidente por sí mismo? La evidencia de este postulado se da, en parte, por que en el adulto, innumerables experiencias han ido formando en él esta convicción, y en parte sobre todo, porque es así el comportamiento de los objetos físicos lo que está al alcance de todos, tanto para el niño como para el hombre común. Es un conocimiento primario acerca de la naturaleza que rápidamente se alcanza, pues múltiplemente se halla esparcido por todo el ámbito de la naturaleza. Estas innumerables experiencias de la coincidencia es lo que permite que se tenga innumerables experiencias desde la época de la niñez en que el hombre se pone en contacto con la naturaleza. Dada la constitución de la inteligencia humana, ésta desde un principio va forjando el concepto de igualdad. Este concepto de igualdad tiene como una de sus fuentes más importantes la coincidencia en el mundo físico. Otra fuente decisiva del concepto de igualdad es la semejanza entre los objetos del mundo físico acercalde la cual el mundofísico ofrece una riquísima experiencia, pues son innumerables los objetos que se presentan como semejantes, semejanza que reposa en la naturaleza física de los objetos mismos, en las características inherentes al objeto mismo. La semejanza tiene que ver con la coincidencia puesto que en la coincidencia, de hecho se superpone un objeto sobre el otro, en tanto que en la semejanza el sujeto cognoscente supone que en el caso que se les superponga, éstos han de coincidir, como de hecho puede acontecer con los objetos en que sea posible hacerlo. Como los objetos semejantes prodigan en la naturaleza, la convicción de la igualdad de lo semejante se da en innumerables oportunidades, desde el comienzo de la niñez, y que el niño lo expresa con la expresión de cosas que se parecen. Es justamente a través de la semejanza que se puede probar que dos objetos son iguales, pues al comparar dos objetos, si se constata que sus características son semejantes, se demuestra al mismo tiempo que son iguales, así como se demuestra que no son iguales cuando al compararlos no se encuentra las suficientes características semejantes. Por todo lo expuesto anteriormente, se comprueba que el concepto de igualdad reposa en última instancia 
en la coincidencia y en la semejanza de los objetos físicos y que la semejanza es una variante de la coincidencia, así como el que el concepto de igualdad tiene que ir precedido de la coincidencia. Entonces el axioma de la coincidencia de Euclides debe de preceder también al axioma de la igualdad el que enuncia que las cosas que son iguales a la misma cosa son también iguales entre sí. De aquí también que estos axiomas no son indemostrables sino que se demuestran basándose en el comportamiento de los objetos físicos mismos, así como el que su evidencia se base en que son conocimientos primarios $y$ que se repitan innumerables veces por hallarse la coincidencia, y su variante de la semejanza, extendida por todo el ámbito de la naturaleza. De los otros dos axiomas de Euclides y que se refiere a que si iguales son añadidos a iguales los todos son iguales; $\mathrm{y}$ que si iguales son substraídos de iguales, la resta es igual, se puede hacer análogas consideraciones y llegar a análogas conclusiones a las referentes a los dos axiomas anteriores. En lo concerniente $a$ la última de las nociones comunes de Euclides, que se enuncia como el que el todo es mayor que la parte, tiene también fundamentalmente como base el comportamiento de los objetos físicos, recogido por la experiencia del sujeto cognoscente, $\mathrm{y}$ que es evidente para el sujeto, por las innumerables experiencias que se le ofrecen, al observar el ámbito de la naturaleza, y se puede comprobar en cualquier oportunidad, que en el mundo físico los objetos siempre se comportan de esa forma. Solamente que es un conocimiento primario que se tiene de la naturaleza, recogido por el hombre desde una temprana edad y de modo tain inmediato, que pasa por desapercibido, o se olvida, que se basa en la experiencia del objeto físico mismo. En resumen, las nociones comunes de Euclides se basan esencialmente en el comportamiento de los objetos físicos, su comprobación de esos mismos objetos, y su evidencia en las innumerables experiencias que se tienen sobre los objetos. Un individuo que careciese de la posibilidad de tener experiencias sobre el ámbito de la naturaleza, no hubiera llegado $\alpha$ esas nociones comunes, ni hubiera podido tener la evidencia de los mismos.

\section{Geometría y universalidad}

En el fondo, la verdad de esas nociones comunes se basa en que así esta constituida la naturaleza y que tales características se manifieston por doquier, lo cual patentiza la universalidad de cómo se manifiestan y la pronta captación por el sujeto cognoscente y lo evidente por sí mismas, así como el que de modo casi inmediato puedan demostrarse auscultando su realidad primaria en el mundo físico. Son las nociones comunes expresión de las ca-

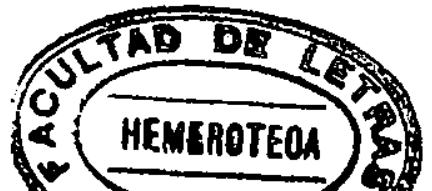


racterísticas universales del ser de las cosas y que conforman la universalidad del pensamiento en cuanto se adecúa a esas características del ser. De aquí que sea completamente falso que la experiencia dé a conocer solamente verdades contingentes, que bien pueden ser o no ser, y que no expresen nada necesario de la naturaleza. La experiencia, por lo contrario, permite conocer aspectos universales y por lo tanto, necesarios de las cosas. La experiencia da a conocer tanto lo universal como lo particular, lo cual por no manifestarse en todas las cosas, sino en algunas de ellas no se manifiesta como necesario y sólo como contingente.

En tanto que en Euclides las nociones comunes se refieren a verdades que son válidas para todas las cosas, sus postulados se refieren a verdades que son válidas únicamente en el área geométrica. De un modo general los postulados son también verdades que no requieren demostración y que son evidentes por sí mismos. Vanos han sido los intentos para demostrar los postulados, pero hay que recalcar que esos intentos entienden por demostración el derivar la verdad de un postulado de otra verdad desde la cual se desprende lógicamente el postulado que se quisiera demostrar. Esto aconteció con el quinto postulado, pero no se le pudo derivar de otro postulado, y por lo tanto se mantuvo intangible e inderivable. En el primero de los postulados se enuncia que se puede trazar una línea recta de cualquier punto a cualquier otro punto. Efectivamente, cualquiera que sea el punto donde partamos podemos realizar lo que enuncia el primer postulado. Es indudable que así enunciado, el postulado no puede derivarse de ninguna otra verdad lgeométrica conocidas $y$ es indudable también que se da como una yerdad evidente por sí misma. Ahora bien, la evidencia del postulado se debe a que en múltiples oportunidades se logra hacerlo, y lo contrario, de no lograr trazar una recta, no se puede hacer. El niño que en la escuela tiene conocimiento por primera vez de este postulado lo capta prontamente porque en su cuaderno de trabajo lo ha hecho muchas veces, así como también en sus caminatas en su medio ambiente, ha podido seguir un sendero recto, así como en cualquier dirección hacia la cual se encaminase. Este postulado tiene su asidero en la experiencia diaria del niño, por la cual rápidamente lo entiende, y se le presenta como evidente por sí mismo. La evidencia del postulado halla su fundamento en la experiencia del individuo adquirida desde muy temprana edad $y$, a su vez, su experiencia proviene del espacio en que se halla sumergido, y de las características que este espacio tiene. Como lo que Euclides buscaba era una verdad geométrica que no se derivase de ninguna otra y que, por lo tanto, le fuese valiosa para fundamentar otras verdades geométricas como los teoremas, como lo enunciado por este primer postulado tenía las características necesarias, lo hizo valer como tal, esto es, 
como postulado. Ahora bien, como no se derivaba de ninguna otra verdad geométrica, se le consideró indemostrable. Es cierto también que Euclides en ningún momento definió lo que era un postulado, que no hizo explícito lo que entendía como tal, pero la forma cómo estructuró sus Elementos, implícitamente daba $\alpha$ en. tender el significado de postulado. Es cierto también que oún cuando daba a entender implícitamente que no eran demostrables los postulados, implícitamente se estaba refiriendo a su indemostrabilidad geométrica, pero en ningún momento se percibe que haya desechado todo tipo de demostración. En ningún momento ha cerrado el camino para emprender una demostración física de los postulados, una demostración que se derivase de las propiedades reales del espacio exterior. Si seguimos el camino que nunca fue cerrado por Euclides, encontramos que los postulados sí son demostrables, aun cuando no demostrable, en sentido estricto, de modo geométrico y lógico. Efectivamente, es una primera demostración física la posibilidad de trazar de hecho una línea recta entre dos puntos de una superficie plana, o la de constatar mediante la observación, que los objetos del mundo circundante poseen puntos materiales visibles extemamente, o invisibles en el interior del objeto pero aprehensibles por la capacidad de imaginación del sujeto, gnoseológicamente legítima y respaldada por los hechos del mundo exterior. Así, en el interior de un objeto material hay muchos puntos materiales que en todas direcciones se pueden conectar en línea recta con otros puntos materiales, $y$ esto es un hecho del mundo físico. De tal modo que la validez del primer postulado de Euclides se demuestra por la naturaleza del espacio exterior en el mundo físico circundante. Por otro lado, no se puede demostrar su validez "partiendo de otra verdad geométrica.

En lo referente al tercer postulado de Euclides en el cual se enuncia que se puede describir un círculo con cualquier centro y distancia, se puede hacer consideraciones análogas $\alpha$ las hechas respecto al primer postulado. En cucnto al cuarto postulado en el que se enuncia que todos los ángulos rectos son iguales el uno al otro, su verdad está respaldada en partes por el principio de coincidencia que como noción común se enuncia por Euclides en la cuarta de ellas: Las cosas que coinciden la una con la otra son iguales la una con la otra. Efectivamente, según Euclides de acuerdo con la definición del ámgulo recto, así lo define: Cuando una línea recta colocada sobre una línea recta hace los ángulos adyacentes iguales el uno al otro, cada uno de estos ángulos es recto, y la línea recta situada sobre la otra es llamada perpendicular a aquella sobre la cual estó situada. En relación con la definición de Euclides se constata que lá igualdad de los ángulos adyacentes es fundamental para determinar las características del ángulo recto. Como hemos visto anteriormente, el concepto de igualdad tie- 
ne una raíz muy importante en la coincidencia física que, como en este ejemplo particular, cada vez que se sitúa un ángulo adyacente sobre el otro, formados según la definición, al superponerlos, se verifica su coincidencia física, esto es, su igualdad. De aquí que el cuarto postulado en cierto modo reitera lo conceptuado en la definición de ángulos rectos, pues esta definición nos habla cuando menos de dos ángulos rectos, los adyacentes. Una vez establecida la igualdad de los adyacentes que también son rectos, esta igualdad es posible referirla al principio de coincidencia. El cuarto postulado no es sino una generalización del criterio de igualdad, el cual en última instancia se apoya en la coincidencia física. Gnoseológicamente, el cuarto postulado, como el primero y el tercero, se fundamenta en el comportamiento de los objetos físicos y en la experiencia que sobre ellos se obtiene. De otro lado, lógicamente se deriva, más bien, de la décima definición del Libro I, en tanto que geométricamente mantiene su independencia de los otros cuatro postulados. El mismo cuarto postulado aclara y refuerza el punto de vista de que la geometría no puede reducirse a un análisis lógico, pues su independencia geométrica se contrapone a su dependencia lógica de una definición, esto es, lógica y geometría no son reducibles la una a la otra. En cambio, la posibilidad de reducir el cuarto postulado a la cuarta noción común de Euclides, la cual se fundamenta en el principio de coincidencia, refuerza la posición de que la geometría es reducible a la física, de que un postulc:do de la geometría no es independiente de la física. Aun cuando tiene la posibilidad de fundamentarse en la física, de derivarsél dél principio de coincidencia, geométricamente no obstante, mantiene su independencia de los otros postulados. Esto se debe a que si bien deriva de un principio físico en general, no puede sin embargo, derivarse de una propiedad física concreta, representada por características geométricas determinadas. A esto se debe su independencia de los otros postulados. Ningún postulado de Euclides puede derivarse el uno de los otros porque cada uno de ellos posee características geométricas propias. Pero si bien esto es verdad geométricamente, no obstante, no excluye, por de pronto de su derivabilidad de una propiedad física más amplia que los postulados de Euclides. Solamente un conocimiento más amplio del mundo físico permitirá constatar que del todo son independientes y por lo tanto, son indemostrables el uno del otro. El distintivo de postulados que han recibido estas cinco propiedades geométricas se ha debido únicamente a que ninguna de ellas es derivable de la otra.

El segundo postulado enuncia que se puede producir una línea recta finita continuamente en una línea recta. Este postulado se presenta como evidente por sí mismo debido a que en innumerables oportunidades es dable ponerlo a prueba en el mundo 
exterior y la experiencia reiteradamente lo comprueba, lo cual, a su vez se debe a que así son las propiedades del espacio exterior. Se resalta, además, que se puede producir una línea recta finita, lo cual indica que Euclides lo pone al alcance aun de nuestra posible experiencia, pues se está refiriendo a un mundo circundante al cual poco a poco vamos alcanzando, o alejóndonos de él. En este postulado, Euclides no hace ningún hipóstasis, ninguna transposición a lo infinito, por lo cual lo circunscribe a nuestra posible experiencia. Lo mismo acontece con el concepto de continuo que el postulado afirma que se pueda trazar una recta. ¿Es este postulado válido? Geométricamente es indemostrable, pero en el espacio físico se puede demostrar su validez $y$, por de pronto, las primeras experiencias parecen confirmarlo. No obstante, la posibilidad de trazar la recta continuamente, aun cuando las primeras experiencias lo confirmen, queda pendiente si es posible definitiva, absolutamente. Solamente el conocimiento del espacio físico podrá comprobar en última instancia si el postulado es justo o no. Su demostrabilidad o indemostrabilidad queda pendiente de un conocimiento mayor del espacio físico, aun cuando geométricamente sea indemostrable.

\section{Indemostrabllidad e inderivabilidad en la geometría}

El quinto postulado, o de las paralelas, atestigua que la verdad evidente por sí misma de los axiomas no era tal, pues si se refiere a la infinitud del espacio, ala verdad del quinto postulado comienza a carecer de fundamentolli Esto es 60 válido únicamente para la experiencia del espacio inmediato, pero en cuanto a su evidencia, si le pretende aplicar más allá, el postulado comienza a perder su evidencia por sí mismo y deja la alternativa de ser sustituido por otros. Esto implica que la característica de evidente por sí mismo de los postulados de Euclides, es porque se refieren a un conocimiento muy restringido, en cierto modo de las propiedades del espacio. La aparente necesidad y universalidad del quinto postulado es en el fondo una ilusión debido a la restricción e inmediatez de las propiedades del espacio proporcionadas por la experiencia.

El quinto postulado de Euclides, conforme él mismo lo enunció, afirma que si una línea recta al cortar dos líneas rectas hace los óngulos interiores del mismo lado, menores que dos rectos, y si ias dos líneas rectas si se continúan, en algún momento se encuentran en el lado en que los ángulos son menores que dos ángulos rectos. Este postulado tan discutido desde la antigüedad griega permitió, finalmente, llegar por su discusión a los planteamientos de la geometría no euclidiana. Los intentos para demostrar este 
postulado desde la época de Tolomeo y Proclo, derivándolo de otro postulado resultaron vanos hasta que, finalmente, en el siglo XIX, con la realización de la geometría no euclidiana se verificó que este postulado era independiente de los otros y por tanto era válida su condición de postulado. Este postulado en el modo en que fue enunciado por Euclides está muy alejado de la experiencia diaria, en parte porque requiere conocimientos geométricos como la suma de los ángulos internos, en parte porque requiere comprender que la menor desviación, por pequeñísima que sea, de la suma de dos rectos de los ángulos internos, ocasionará que las rectas se encuentren en algún punto del espacio. Pero, sobre todo, requiere la comprensión del concepto de líneas rectas producidas indefinidamente. Este concepto de indefinido obliga a proyectarse en distancias del espacio que escapa a toda imaginación cercana a la experiencia, y por eso en la experiencia diaria no se encuentra ningún asidero para aceptarlo con evidencia. Bajo este aspecto no es un postulado que pueda ser calificado como evidente por sí mismo. Si el postulado es una proposición que no requiere demostración y es evidente por sí mismo, el quinto postulado desmiente que ésta sea una de sus características, pues no resulta evidente por sí mismo. En cambio sí se manifiesta como una proposición cuya verdad no puede ser demostrada. De tal modo que la evidencia por sí misma y la no demostrabilidad no son características que necesariamente tengan que darse conjuntamente. La evidencia por sí misma es un estado del conocimiento al cual se llega cuando una verdad es fácilmente aprehensible y se debe a que la experiendia cotidiana rápidamente la constata y verifica. Por lo menos, éste es elpcaso de algunas definiciones y postulados de la geometría. El postulado geométrico, en cambio, es una verdad que no puede ser demostrada, aun cuando no sea fácil de constatar ni sea evidente por sí mismo, como acontece con el quinto postulado en el modo como lo enunció Euclides. La indemostrabilidad del postulado, además, se debe fundamentalmente a que es un principio, y que como tal, más allá de él no se puede continuar. A su vez, como postulado geométrico, es la base que sustenta y explica otras verdades geométricas. De tal modo que la cuestión de que un postulado sea evidente por sí mismo no tiene importancia alguna, pero es probable que los antiguos como Tolomeo y Proclo, al no hallar la evidencia por sí misma del quinto postulado supusieran que había que desecharlo como postulado a intentaron su demostración. En la discusión que en numerosas ocasiones suscitó el quinto postulado se llegó a una enunciación clara en el modo como lo hizo Playfair en el sigo XVIII, recogiendo otras enunciaciones semejantes anteriores. El modo de Playfair, que ha quedado definitivamente como el.verdadero modo se enuncia así: A través de un punto dado en un mismo plano se 
puede trazar solamente una línea paralela a una línea recta dada. Ciertamente que este modo de enunciar el quinto postulado resulta más claro y simple que el de Euclides y por lo cual se manifiesta como evirente por sí mismo. Ahora bien, a qué se debe esta evidencia, sulo es al hecho de que cuantas veces tracemos una línea recta y un punto situado fuera de ella, jamás lograremos trazar más de una paralela a la recta dada a partir del punto dado. Es en nuestra experiencia inmediata, cuando en innumerables ocasiones logramos trazar solamente una paralela, en que se basa la convicción de la verdad de este postulado y su carácter de evidente por sí mismo. No obstante, a pesar de presentarse como evidente por sí mismo, su condición de postulado no fue definitivo sino en el siglo XIX, cuando aparece la geometría no euclidiana de Lobachevsky y Bolyai, quienes al eliminar el quinto postulado de Euclides y Playfair y sustituirlo por otro: Por un punto dado se puede trazar más de una línea recta paralela a una recta dada, lograron hacer comprender que el quinto postulado no dependía de los otros cuatro, que no se derivaba de ellos, que era indemostrable a partir de otras verdades geométricas, y que por lo tanto, era válido su condición de postulado al lado de los otros cuatro. Esto nos comprueba de manera fehaciente que el carácter de evidente por sí mismo y de indemostrabilidad de un postulado son dos aspectos distintos, puesto que este último postulado es indemostrable pero no evidente por sí mismo. Recalquemos que la indemostrabilidad de los postulados de Euclides, solamente significa su inderivabilidad de otras verdades geométricas, pero de ninguna manera su indemostrabilidad de las propiedades del espacio físico.

\section{Geometría pura o matemática y geometría aplicada o física}

La geometría clásica o euclidiana había tácitamente aceptado que la geometría se refería a propiedades del mundo físico. Con el advenimiento de las geometrías no euclidianas, que pusieron en tela de juicio la validez de los axiomas y teoremas de la geometría euclidana, los científicos y filósofos retiraron su adhesión incondicional a los planteamientos de Euclides y surgieron dudas acerca del respaldo que el mundo físico podría dar a los principios enunciados por la geometría euclidiana. Pero, por otro lado, era también un hecho que la geometría euclidiana había tenido su aplicación en el mundo material. No se necesitaba entonces mayores pruebas de que la geometría, tal como la entendieron los clásicos, se refería al espacio del mundo físico. El advenimiento de las geometrías no euclidianas hubiera debido llevar solamente a la convicción de que la geometría euclidiana representaba sola- 
mente un campo limitado de las propiedades del espacio y que las nuevas geometrías abarcaban un campo mayor. Esta convicción no hubiese sido inusitada en la historia de la ciencia, puesto que muchas teorías o leyes más amplias de una disciplina científica, aun cuando han substituido como concepción general de las anteriores, muchas veces han dejado en pie, la parte de verdad que encerraban las anteriores. Pero con la geometría el curso de la historia está siguiendo otro camino, o pretenden seguir otro camino algunos de los científicos y filósofos, cuya posición se manifiesta en aceptar como válidas tanto la geometría euclidiana como la no euclidiana $\mathrm{y}$ en sostener que en última instancia ninguna de ellas se refiere a propiedades reales del espacio. ¿Por qué se llegó a esta posición?

En primer término, porque ambas eran lógicamente aceptables, puesto que todas las propiedades contenidas en cada una de ellas, se podían derivar deductivamente de ciertos enunciados generales. En segundo lugar, porque el geómetra había podido construirlas sin recurrir a la experiencia, y por lo tanto sin un estudio del espacio físico, lo que permite suponer, naturalmente, de que no se refiere la geomertía al espacio real. Sin referirse a un espacio físico y teniendo en cuenta únicamente la validez lógica de las conclusiones, el geómetra considera que la geometría es una ciencia pura o matemática. Mas, el físico no parece muy satisfecho con esta solución dada al problema de la validez de la geometría, e insiste en la necesidad de contar con una geometría que se refiera al espacio del mundo físico con el que tiene que tratar, y no considera entonces adecuado que cualesquiera de las geometrías sean igualmente válidas $y$ exige que los principios de la geometría tengan su respala en elmundolísice, y de que su validez deba someterse en última instancia a la prueba de la experiencia. La geometría debe ser entonces una ciencia física o aplicada. De aquí que en la actualidad se distinga la geometría pura o matemá. tica de la geometría aplicada o física. ¿Es acaso justificado man. tener estas dos posiciones?

El mismo quinto postulado de Euclides, que con el advenimiento de la geometría no euclidiana del siglo XIX, al ser substituido por el quinto postulado de Lobachevski y Bolyai, según el cual se puede trazar más de una recta paralela a otra recta o partir de un punto dado fuera de ella; o por el postulado de Riemann, según el cual no se puede trazar ninguna recta paralela a una recta dada a partir de un punto fuera de ella, deja en una encrucijada a la geometría en cuanto a cuál de los tres postulados es concorde con la realidad de las cosas, con la realidad del espacio exterior. Hay dos maneras de salir de esta encrucijada. La primera de ellas consiste en aceptar como válidos los tres postulados y las consecuencias que esto implica para los teoremas de la geometría misma; y la se- 
gunda como aceptar como válida una de las tres formas de la geometría solamente. Estas dos maneras de abordar las verdades geométricas llevó a distinguir dos tipos de geometría: la geometría pura o matemática y la geometría aplicada o física. Sin embargo, la aceptación de dos tipos de geometría no permite llegar a los fundamentos de la geometría misma. Así, en el caso de aceptar las tres formas de geometría, la geometría se justifica únicamente por sus fundamentos lógicos, esto es, la geometría pura o matemática se apoya en última instancia en la lógica geométrica; en el caso de aceptar solamente una de las tres formas de la geometría, la geometría reposa definitivamente en los fundamentos del espacio exterior, esto es, la geometría aplicada o física se apoya exclusivamente en la naturaleza del espacio físico. Aun cuando para hallar una salida inmediata a la encrucijada sea aceptable el conservar los dos tipos de geometría; pura o matemática y aplicada - física, esta aparente solución no resiste a un análisis filosófico de los fundamentos verdaderos de la geometría. El aceptar los dos tipos de geometría sería aceptar, por un lado, que el espacio es una concepción lógica, y aceptar, por otro lado, que el espacio es un hecho físico, lo cual implicaría una incongruencia gnoseológica, pues el espacio sería tanto una mera representación mental como un hecho físico de la realidad exterior. Estos dos criterios no pueden aceptarse como válidos $\mathrm{y}$, más bien, el uno excluye al otro, o de lo contrario, se trata de dos disciplinas esencialmente diferentes y que solamente en la apariencia son la misma. Efectivamente, la geometría pura o matemática parte, como pretende, de ciertos axiomas, que no tienen que ser fundamentados en la realidad exterior, a partir de los cuales va a deducir lógicamente los teoremas geométricos en tanto que la geometría física o aplicada, sí requiere necesariamente que sus axiomas se hallen respaldados por la naturaleza de la realidad exterior, y por lo tanto el primer tipo de geometría y el segundo no tienen por qué coincidir, y ser igualmente válidos. Para mayor exactitud, digamos que son igualmente válidos en tanto que la geometría pura o matemática recibe su validez exclusivamente de la estructuración lógica de todo el sistema y, en cambio, la geometría física o aplicada recibe su validez tanto de la naturaleza del espacio físico exterior, como también de la ordenación lógica de sus deducciones. Así la diferencia entre los dos tipos de geometría no estriba en la estructuración lógica, pues ambas la poseen, sino en el necesario respaldo que debe dar la naturaleza del espacio físico o exterior, o el prescindir de esta última exigencia. Queda entonces por indagar si la validez última de la geometría se debe en parte a la estructuración lógica o si es necesario fundamentarla en todos los casos en el espacio físico. En verdad, detrás de todo axioma de 
la geometría hay referencia a una relación espacial, aun cuando sea de modo muy abstracto o generalizado.

\section{Lógica y geometría}

Cuando el geómetra indaga, con toda justeza, en la lógica que hay en cada axioma lo único que hace es destacar la relación lógica con el axioma. Si afirmase que esta relación lógica es el fundamento de la geometría estaría confundiendo lógica con geometría, pues efectivamente, la lógica del axioma geométrico no es exclusividad de este axioma sino que se halla en cualquier otra disciplina como la física, la biología, la psicología y las ciencias sociales. Si el geómetra, dijese que la geometría es en última instancia lógica debería también decir que todas las disciplinas, como las que se acaban de enumerar, son también fundamentalmente lógicas. Si así lo dijese, el geómetra caería en un logicismo respecto a todas las ciencias, no solamente las matemáticas, sino también a todas las demás ciencias, ya sean físicas, biológicas y humanas. Sería como caer en una modalidad del idealismo filosófico que bien pudiera llamarse idealismo logicista. Contrariamente a este logicismo, lo indícado es sostener, al comprobar la vigencia de lo lógico en toda la naturaleza, que lo lógico expresa una relación de todas las cosas del mundo exterior, o de la naturaleza misma, y que no es una simple arbitrariamente 0 arbitrariedad de la mente humana. Consideremos algunos axiomas de la geometría en relación a lo que se sostiene en las últimas líneas.

Es evidente quile los postulddoś de Euclides se refieren a relaciones espaciales y que Euclides implícitamente da por supuesto que son las reláciones del espacio físico exterior. De igual modo. el postulado de las infinitas paralelas o más de una paralela a una recta dada de Gauss, Lobachevski y Bolyai, expresan también relaciones espaciales que se refieren a un espacio físico exterior, como de hecho Gauss intentó demostrar trazando un triángulo con las cumbres de tres montañas para verificar si divergen sus ángulos de la suma de dos rectos, o cuando Lobachevski sostiene que en el paralelaje de las estrellas se comprobará que los ángulos de un triángulo son menores que dos rectos. Igualmente, el postulado de ninguna paralela a una recta dada de Riemann se refiere a relaciones espaciales. Einstein muy bien comprendió que eran vólidas para un mundo físico y por este motivo se apoyó en la geometría de Riemann para sus concepciones cosmológicas. Así mismo, la geometría de Hilbert intenta con sus postulados dar un nuevo fundamento a la geometría de Euclides y es también evidente que estos postulados se refieren a las relaciones espaciales del espacio exterior, y es por esto, que el mismo postulado de Playfair está incluido en los postulados de Hilbert. 
Consideremos ahora cómo expresar lógicamente los postulados de Euclides. En el primero se dice que entre dos puntos cualesquiera se puede trazar una línea recta. Esto quiere decir que por dos puntos distintos hay una línea recta a la cual pertenece cada uno de ellos. Si enunciamos de nuevo este postulado en una forma esquemática, puede cambiarse de este modo: Para cualesquiera dos $\mathrm{P}$ distintos, hay un $\mathrm{S}$ con el cual cada uno de ellos mantiene la relación B. Aquí en lugar de puntos hablamos del concepto lógico $\mathrm{P}$, y en lugar de hablar de dos líneas rectas hablamos del concepto lógico de $\mathrm{S}$, y en lugar de una cosa perteneciente a otra hablamos de un concepto lógico que mantiene la relación B con otro.

Esta manera de enunciar lógicamente un postulado geométrico es muy justificado, por cuanto permite constatar las relaciones lógicas que hay en la enunciación de un postulado. Sin embargo, esta manera no interpretada de enunciar lógicamente una relación, permite darle una manera interpretada de enunciación, no solamente en el modo interpretado del postulado de Euclides, sino también de otras relaciones geométricas como por ejemplo: Para cualesquiera dos líneas rectas distintas, hay un punto en el cual ambas de ellas terminan; o para cualesquiera dos instantes distintos, hay un intervalo al cual pertenece cada uno de ellos. (Estos dos ejemplos anteriores de Barker). Ahora bien, al lado de estas dos maneras concretas de expresar el enunciado lógico, hay innumerables maneras de expresar concretamente el mismo enunciado lógico. Así, es también legítimo decir: Para dos estudiantes cualesquiera hay una universidad a la cual cada uno de ellos pertenece; para dos hermanos cualesquiera hay una relación de mayorazgo a la cual cada 'cuno de ellos perteneée:" para cualquiera de dos ondas electromagnéticas hay una relación de intensidad a la cual cada uno de ellos pertenece; para cualquiera de las raíces de dos plantas hay una relación de geotropismo a la cual cada una de ellas pertenece; para cualquiera de dos personas alegres hay un motivo de alegría a la cual cada una de ellas pertenece. Estas múltiples maneras de concretarse la enunciación lógica indica que esa misma relación lógica no es propia de la geometría sino una relación lógica que como tal es independiente de su manera concreta. Por lo tanto, si se pretendiese reducir la geometría al enunciado lógico, también sería entonces justificado reducir todas las ciencias a la lógica y decir que en última instancia no solamente la geometría, no solamente todas las matemáticas, sino también todas las ciencias se reducen a las relaciones lógicas. Por este camino se llegaría a una especie de idealismo lógico total, en el cual parcialmente caen los que sostienen que las matemáticas son relaciones lógicas, como la afirma Bertrand Russell. En verdad, el haber descubierto las relaciones lógicas 
que se da en cualquier postulado de la geometría o de las matemáticas, solamente significa que nada en la naturaleza está libre de esa relación, por lo cual lo lógico es la expresión de las relaciones primordiales de todas las cosas; pero es algo muy diferente que las relaciones lógicas sean principios que se encuentran en todas las cosas, que decir que estas relaciones lógicas existen por sí mismas. Las relaciones lógicas no existen independientemente de las cosas concretas de la naturaleza, sino que son sus leyes más generales, y no pueden existir por sí mismas, como tampoco existen por sí mismas otras leyes de la naturaleza, como por ejemplo, la ley de la gravitación, no existe por sí misma sino que es una ley que se da en cuanto hay objetos materiales que son atraídos los unos a los otros. De aquí que no puede haber geometría sin relaciones espaciales y sin espacio físico exterior, como tampoco puede haber física sin cuerpos materiales, ni biología sin seres vivos existentes, ni psicología sin hombres o animales con estados psíquicos. No hay leyes que existan sin las cosas concretas de la naturaleza, ni tampoco principios lógicos sin esos mismos objetos concretos de la naturaleza. No hay principios o relaciones lógicas sin la existencia de objetos concretos de la naturaleza. Afirmar lo contrario es no distinguir entre la capacidad abstractiva del hombre y la existencia concreta de las relaciones que descubre, como también implicaría no haber distinguido entre la misma capacidad abstractiva del hombre y las leyes de la física, o de la biología, por ejemplo, que las ha descubierto en las relaciones de las cosas existentes. Sería sostener que el principio de las cosas de la naturaleza existe pör sí mismo.

\section{Abstracción geométrica y espacio físico}

\section{"Jorge Puccinelli Converso"}

Las propiedades geométricas tendrán que conocerse siempre auscultando la naturaleza del espacio físico exterior, en el cual se fundamentan estas propiedades. Sin embargo, ¿cómo es posible que el geómetra pueda, con su sola abstracción mental, llegar al conocimiento exacto de las propiedades geométricas, sin tener que recurrir a una indagación sobre el espacio físico, midiendo o experimentando acerca de las relaciones espaciales? Esta independencia de la mente del geómetra de la realidad física exterior es más aparente que real. La geometría empírica de los egipcios y babilonios nos recuerda que es auscultando sobre el terreno mismo que descubrieron una serie de propiedades geométricas. Ahora bien, cuando el geómetra traza sobre una tabilla o sobre el papel una figura geométrica, sigue trazando sobre ese mismo espacio físico las mismas relaciones geométricas que ha trazado sobre el terreno, puesto que las líneas trazadas en el papel lo son en el espacio 
físico. Por otro lado, la capacidad de la mente humana de idear una serie de figuras geométricas se debe a que la naturaleza exterior es pródiga en figuras geométricas, por las variadas formas como se presentan los objetos de la naturaleza, como al hecho físico que el espacio se da con sólo tres dimensiones, lo que permite que la mente humana pueda combinar con relativa facilidad estas tres dimensiones espaciales entre sí para configurar innumerables figuras geométricas, que la naturaleza quizá no haya utilizado, para dar forma a sus objetos, por lo menos en el mundo macroscópico, aun cuando a escala microscópica parecen ser inagotables las formas de la naturaleza. La mente humana tiene la capacidad para combinar esas tres únicas dimensiones del espacio que son las que se ofrecen en su experiencia cotidiana. No obstante, queda en el marco de la simple suposición, las tremendas dificultades en que se hallaría la mente humana para conformar figuras geométricas, si por hipótesis hubiera una región del espacio que poseyera múltiples dimensiones. Para esto la mente humana no está desarrollada y su capacidad abstractiva tropezaría con considerables dificultades para desarrollar una geometría basada en la pura abstracción mental. En verdad la capacidad abstractiva del geómetra es posible por la simplicidad de las tres únicas dimensiones del espacio como se presentan en la experiencia. ¿Cómo se explica entonces el espacio de n-dimensiones creado por las matemáticas? En verdad, el espacio de n-dimensiones es solamente una generalización del espacio de tres dimensiones. Las n-dimensiones más allá de tres dimensiones, no tiene propiamente contenido espaciaby expresan una generalización algebraica exclusivamente. De aqui que dentro de este marco estrictamente algebraico no se ha "elaboradocninguna geometría de n-dimensiones, pues, por un lado, ni el matemático puede representarse un espacio cuatridimensional o de más dimensiones, puesto que su mente no se ha conformado sino al contacto con un espacio tridimensional, ni tampoco el matemático ha demostrado ni verificado la existencia de un espacio de más de tres dimensiones. Por eso, desde el punto de vista gnoseológico, en cuanto se refiere a la percepción y representación mental respectivamente, no se puede rebasar el espacio de tres dimensiones, porque así únicamente de modo tridimensional es dado el espacio físico; ni tampoco es posible elaborar una geometría de más de tres dimensiones porque no hay espacio físico que lo respalde. Esto no quiere decir que es imposible elaborar una geometría de más de tres dimensiones, sino que solamente será posible elaborarlo en el caso de que la ciencia descubra que el espacio físico posee más de tres dimensiones. En última instancia la geometría ha de apoyarse necesariamente en la realidad del espacio físico. 
Las características del espacio son recogidas a través de la experiencia del espacio exterior. ¿Por qué los enunciados geométricos se dan con el carácter de universalidad y necesidad? Los teoremas de Euclides, Lobachevski y Riemann se presentan cada uno de ellos con esas características, si se precisa como lo hiciera Beltrami que cada una de estas geometrías es válida, según Beltrami, para las superficies planas, esféricas y pseudoesféricas respectivamente. La geometría euclidiana permitía concluir que la to espacial como por el aspecto gnoseológico que se apoyaba en aquél. El advenimiento de la geometría no euclidiana permitió distinguir entre la universalidad y necesidad basadas en el comuniversalidad y la necesidad se debía tanto por el comportamienportamiento espacial, de la universalidad y necesidad gnoseológicas. Pues en tanto que la universalidad y necesidad espaciales de la geometría de Euclides fue socavado por la nueva geometría, no sucedió así con la necesidad y universalidad con que se presentaban las nuevas geometrías, en el aspecto gnoseológico. Esto se debe a que partiendo de un enunciado diferente del postulado quinto de Euclides, se obtienen lógicamente las conclusiones no euclidianas. Ahora bien, cualquier deducción lógica también se presenta con las características de universalidad y necesidad y de aquí que las nuevas geometrías se presentasen con las mismas características por su fondo lógico. Lo que aconteció con la geometría euclidiana es que la referencia al espacio físico que se con. sideraba con la cadractetísticas de unitversalidad y necesidad, estaba acompañado con las mismas características, pero de origen lógico. Al replantearse el 'quinto postufado, la geometría mantuvo su necesidad lógica, pero quedó en suspenso su necesidad referente al espacio, no obstante que los fundadores de la nueva geometría insistían en que eran aplicables al espacio físico. Sin embargo, esta conjunción de lo universal y necesario espacial y lógico en la geometría euclidiana incrementaba considerablemente la convicción de su validez, en tanto que el advenimiento de la geometría no euclidiana, que permitió la escisión de lo espacial y lo lógico, ha llevado, a nuestro parecer equivocadamente, a algunos geómetras, intentar reducir la geometría a la lógica, por no haber diferenciado debidamente la conjunción de la universalidad y necesidad espacial y lógica.

De otro lado, el advenimiento de la geometría no euclidiana nos permite constatar que universalidad y necesidad son dos características que no están indisolublemente vinculadas, pues si bien todo lo universal es necesario, no todo lo necesario es universal. $\mathrm{Si}$ el espacio, por hallarse en todo el universo físico, es entonces universal, y si es homogéneo e isotrópico, entonces las relaciones 
- propiedades espaciales también serán universales así como necesarias. Esta universalidad y necesidad no es prerrogativa del espacio euclidiano sino también el no euclidiano: de la superficie plana, esférica o pseudoesférica. Ahora bien, poseen estas características en tanto que en cualquier lugar del espacio es posible establecer las mismas relaciones espaciales de forma. Pero en lo que se refiere a las magnitudes, las propiedades varían según las dimensiones de las figuras que se considere, como sucede, por ejemplo, con las relaciones de magnitud en la suma de los ángulos de un triángulo. De aquí que las propiedades espaciales sean universales en cualesquiera de las tres geometrías consideradas, pero las relaciones de magnitud son diferentes según el área considerada, y por este motivo, estas relaciones de magnitud no son universales, pero si son necesarias. En este sentido, el apriorismo gnoseológico del conocimiento geométrico queda rebasado por cuanto no subsiste el criterio de universalidad, pero no por el de necesidad, el cual se mantiene. Es exclusivamente a la no subsistencia de la universalidad que carece de validez el neoapriorismo gnoseológico, en lo que respecta al espacio, y resultan por tanto vanos los intentos de restablecerio.

Las propiedades del espacio físico, la homogeneidad y la isotropía, son pues las que fundamentan, a su vez, los mismos criterios de los juicios geométricos. Ahora bien, ¿a qué se debe que la mente humana pueda ella misma emitir juicios universales y necesarios sobre las relaciones espaciales geométricas? Se debe a nuestro parecer, en parte a la homogeneidad e isotropía del espacio, y en parte también a quécel espacio físico es relativamente simple, pues posee únicamente tres dimensiones. La tridimensionalidad permite que la mente elabore adecuadamente sus conceptos sobre el espacio. Si éste tuviese innumerables dimensiones, la tarea sería enormemente más considerable. Resulta relativamente simple para el hombre conforme repetidamente las mismas figuras geométricas y representárselas mentalmente. Esta relativa simplicidad es la que permite una satisfactoria adecuación entre la representación mental y el espacio físico. Por otro lado, esta misma simplicidad puede suscitar la ilusión de que las propiedades del espacio y de las figuras geométricas son creaciones exclusivas de la mente humana, ajena a la realidad exterior. Además, como en cualquier lugar del espacio es posible trazar figuras simples, como en verdad son las que constituyen la geometría euclidiana y no euclidiana, esto también contribuye a la ilusión de que es la mente la creadora de la geometría, siendo en verdad debido a que la tridimensionalidad del espacio, su homogeneidad e isotropía, lo que nos ofrece la posibilidad de actuar sobre él indefinidamente. En cambio, en lo que respecta a las figuras complejas y aún caprichosas, que es posible trazar en el espacio, aún se 
hallan en estudio, $y$ se seguirán trazando $y$ estudiando nuevas figuras geométricas. El espacio es maleable y permite que sobre él se trace desde las figuras más simples hasta las más complejas y la mente humana ha descubierto todas estas posibilidades que le ofrece el espacio. Pero ha descubierto sólo la posibilidad, y sólo ha conocido hasta ahora las propiedades de las figuras más simples.

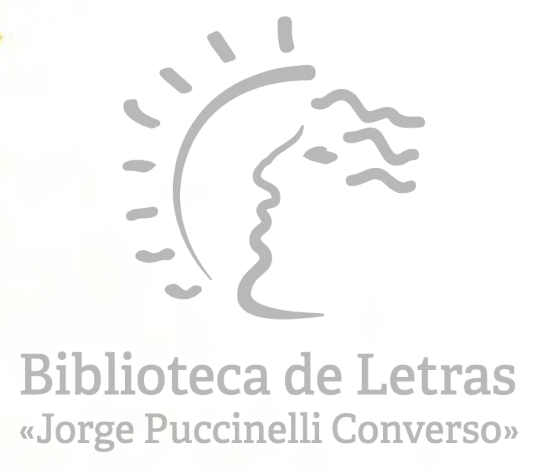

